\title{
四川盆地降水过程中水气之间 $\mathrm{SO}_{2}$ 浓度关系
}

\author{
雷孝恩袁素珍 \\ (中国科学院大气物理研究肪, 北京)
}

Drewes $^{[1]}$ 在 SMICK 模式中,已将大气化学动力学和大气输送扩散过程联合起来, 提出 了在降水过程中污染物液相和气相浓度的两类控制方程,在实际应用中获得了好的结果,并指 出液相浓度受气体浓度的影响. $\mathrm{Pena}{ }^{[2]}$ 研究过降水中 $\mathrm{SO}_{2}$ 含量与大气中浓度之间关系, 并 分析了 $\mathrm{SO}_{2}$ 与降水中 $\mathrm{pH}$ 的关系. $\mathrm{Hales}^{[3]}$ 利用 $\mathrm{SO}_{2}$ 液相和气相浓度平衡关系, 由已知气体 浓度计算水中 $\mathrm{SO}_{2}$ 含量,并和实际作了比较.

本研究则是在综合分析四川盆地同步取得的 $\mathrm{SO}_{2}$ 浓度, 雨水酸度 $\mathrm{pH}$, 温度以及降水分布 资料的基础上,应用 $\mathrm{SO}_{2}$ 液相和气相浓度平衡关系, 导出适用于四川盆地 $\mathrm{pH}$ 与 $\mathrm{SO}_{2}$ 浓度之间 经验关系,为我国最严重的酸雨污染区的诊断,预测及控制提供适用的方法.

\section{一、 $\mathrm{SO}_{2}$ 浓度变化与降水过程的关系}

Fisher ${ }^{[4]}$ 在研究降水系统的 $\mathrm{SO}_{2}$ 迁移和输送过程中指出, 雨水酸庶 $\mathrm{rH}$ 在 4-5 范围内的


大气中 $\mathrm{SO}_{2}$ 浓度之间存在有负相关. 因此, 降水过程中 $\mathrm{SO}_{2}$ 浓度变化规浡的认识对相应的雨 水中酸度的确定是非常重要的.

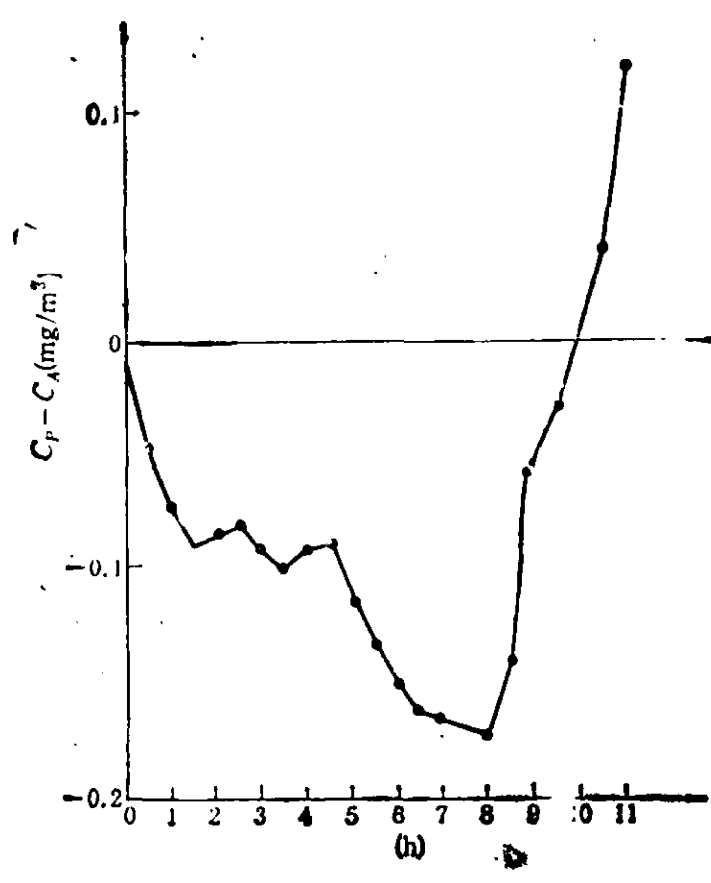

图 1 重庆 $C_{P}-c_{A}$ 随时间怒化

$C_{P}$ 代表降水过程 $\mathrm{SO}_{2}$ 浓度; $C_{A}$ 代 80 ，总体中均浓度



目 2 江油 $C_{P}-C_{A}$ 随时间变化

$C_{P}$ 与 $C_{A}$ 说明如图 1

本文 1986 年11月27日收到。 
为了分析这一变化规律, 我们在四川盆地进行了一系列降水化学和 $\mathrm{SO}_{2}$ 浓度的同步自动 化测量, 这里只给出重庆(代表重酸雨区)和江油(代表轻酸雨区)两个站的观测结果.

重庆 $y$ 月 22 日点到 11 点的 $C_{P}-C_{A}$ 随时间变化曲线(图 1) 表明,降水一开始

$$
C_{P}-C_{A}<0 \text {, }
$$

到降水停止后, $C_{P}-C_{A} \geqslant 0$, 即 $\mathrm{SO}_{\mathrm{i}}$ 浓度立刻回升。这一现象在 1985 年 9 月 25 日的江 油 站（图 2) 也表现得非常明显, 其分布规律与重庆一样, 只是江油 $\mathrm{SO}_{2}$ 浓度比重庆低, 因而 $C_{P}-C_{A}$ 的绝对值比重庆小, 即降水清洗作用也小，表明清洗作用的大小与大气中 $\mathrm{SO}_{2}$ 浓度 本身有密切关系.

\section{二、 $\mathrm{SO}_{2}$ 液相和气相浓度平嘼关系}

由于 $\mathrm{SO}_{3}$ 气体在大气中有相当强的湍流扩散能力, 因而气相和液相之间物质的交策是相 当快的. 野外试验表明,平衡清洗条件在 $\mathrm{SO}_{2}$ 污染源附近不满足，但对于区域性 $\mathrm{SO}_{2}$ 清洗过程 的计算是满足的. 本文关心的是四川盆地酸雨研究，完全可以使用平衡清洗条件来导出我们 所需要的关系. Hales ${ }^{[3]}$ 给出了这种情况液相 $\mathrm{SO}_{2}$ 浓度 $C_{x}$ 的如下关系:

$$
C_{x}-\frac{C_{y}}{H}+\left\{-\left[\mathrm{H}_{3} \mathrm{O}^{+}\right]+\left(\left[\mathrm{H}_{3} \mathrm{O}^{+}\right]^{2}+4 K_{1} C_{v} / H\right)^{\frac{1}{2}}\right\} / 2,
$$

这里 $\left[\mathrm{H}_{3} \mathrm{O}^{+}\right]$(克分子量/升)表示贡献给降水的氢离子浓度,作为第一近似,它与 $\mathrm{pH}$ 有如下关 系:

按 Pena ${ }^{(2)}$ 研究结果

$$
\left[\mathrm{H}_{3} \mathrm{O}^{+}\right]-10^{-\mathrm{PH}},
$$

$$
C_{x}-A C_{y},
$$

其中 $A$ 为可溶参数, $C y$ 为大气中 $\mathrm{SO}_{2}$ 浓度 (克分子量/升), 将 (2) 和 (3) 式代人 (1), 则

$$
C_{y} A-\frac{C_{y_{-}}}{H}+\left\{-10^{-\mathrm{pH}}+\left(10^{-2 \mathrm{pH}}+4 K_{1} C_{y} / H\right)^{\mathrm{L}^{2}}\right\} / 2,
$$

其 $A$ 给成

$$
A=K_{H}\left[1+K_{1} / 10^{-\mathrm{pH}}\right] \cdot F_{0},
$$

$K_{H}, K_{1}$ 和 $H$ 是绝对温度 $T$ 的函数, 其值分别由以下表达式给出:

$$
\begin{aligned}
& K_{H}=\exp \left[\frac{3163.52}{T}-9.576\right], \\
& K_{1}=\exp [2.34-0.0224 T], \\
& H=1.015 \times 10^{-25} T^{9.499},
\end{aligned}
$$

$F_{0}$ 是一个待定的经验常数.

\section{三、 $\mathrm{SO}_{2}$ 浓度与 $\mathrm{pH}$ 的经验关系}

关系式 (4) 看起来很不直观, 实用起来很不方便,为此,我们用数值逼近法在给定不同的 $\mathrm{SO}_{2}$ 浓度, $\mathrm{T}$ 和 $\mathrm{pH}$ 情况下, 对 (4) 式进行了详细的数值计算, 由这些理论计算的结果, 再用多 元非线性回归方法, 发现包含三个因子(即 $C_{y}, T$ 和 $\mathrm{pH}$ ) 的表达式 (4) 可用以下关系等效表 示:

$$
\mathrm{pH}=\left(A_{0}-B_{0} \ln T\right) C_{y}{ }^{-0.04817},
$$


(9) 式比 (4) 式清䞨直观得多, 其中 $A_{0}$ 和 $B_{0}$ 是依赖 $F_{0}$ 的常数.

关系式 (4) 或 (9) 是在某些假设情况下得到的,实际大气不会完全满足这些条件, 尤其是 雨水中的 $\mathrm{SO}_{2}$ 与 $\mathrm{pH}$ 的关系还步及到一些复杂的降水化学和大气化学过程, 在这些过程尚没 有弄清楚之前, 为实际需要, 作经验订正是可行的, 即虽然一些复杂过程我们不清趋, 但它最后 的结果是知道的. 为此, 我们使用四川盆地范围内 $\mathrm{SO}_{2} 、 \mathrm{pH}$ 、降水量和温度同步观测资料, 对 (9) 式中的经验参数 $A_{0}$ 和 $B_{0}$ 进行订正, 其经验订正的具体归结到待定的参数 $F_{0}$ 上, 根据野 外实测资料, 对不同的观测站, $F_{0}$ 的值不同 (表 1). 与表 1 中 $F_{0}$ 相对应的 $A_{0}$ 和 $B_{0}$ 如表 2 所示.

表 1 各观测站的 $F_{0}$

\begin{tabular}{c|c|c|c|c|c|c|c|c|c|c}
\hline 站名 & 成都 & 量庆 & 万县 & 乐山 & 内江 & 宜宾 & 自页 & 南充 & 玚阳 & 垱州 \\
\hline$F_{0}$ & 1.94 & 7.63 & 6.21 & 2.93 & 3.82 & 1.76 & 6.67 & 4.79 & 2.79 & 3.48 \\
\hline
\end{tabular}

表 2 (9) 式中的常数值

\begin{tabular}{c|c|c|c|c|c|c|c|c|c|c}
\hline 站名 & 成都 & 重庆 & 万县 & 乐山 & 内江 & 宜宾 & 自定 & 南充 & 线阳 & 洲 \\
\hline$A_{0}$ & 49.71 & 43.29 & 45.03 & 48.35 & 47.36 & 51.64 & 43.99 & 45.94 & 48.73 & 48.05 \\
\hline$B_{0}$ & 7.91 & 6.89 & 7.17 & 7.7 & 7.54 & 8.22 & 7.0 & 7.31 & 7.76 & 7.65 \\
\hline
\end{tabular}

关系式 (9) 是否符合实际,或与实际符合到什么程度,这要看它用到酸雨模式中实际预测 的效果来决定. 从我们已将关系式 (9) 用到对重庆和四川盆地酸雨 ${ }^{[5,6]}$ 分布的实际沴断效果 来看,其结果是令人鼓舞的.

\section{四、小结}

通过以上分析,我们可作如下小结:

降水期间大气中 $\mathrm{SO}_{2}$ 浓度显著减小,表明降水对 $\mathrm{SO}_{2}$ 气体清洗作用是明显的, 雨水中的酸 度 $\mathrm{pH}$ 值与大气中 $\mathrm{SO}_{2}$ 浓度有密切关系, $\mathrm{SO}_{2}$ 浓度越高, 其清洗的量也越大.

利用水中和气体中 $\mathrm{SO}_{2}$ 的浓度平衡理论，本文导出了经过实测资料校正的 $\mathrm{pH}, \mathrm{SO}_{2}$ 浓度 和温度之间的经验关系,它可供酸雨模式计算时使用.

对实际的大气情况，虽然 $\mathrm{pH} 、 \mathrm{SO}_{2}$ 浓度、温度以及其它尚未考虑因子之间的关系是非常 复杂的,本文的结果只是初步的,而且具有明显的经验性，有关这方面的工作尚需继续深人研 究,但作为认识的第一步,本文的结果与实际有这样好的一致性是可喜的.

[1] Drewes, D. R., Atmospheric Environment, 16 (1982), 1717-1724.

[ 2 j Pens, J. A., Atmospheric Environment, 16 (1982), 1711-1715.

[3] Hales, J. M., Atmospheric Environment, 13(1979), 1121-1132.

[4] Fisher, B. E. A., Atmospheric Environmint, 16 (1982), 775-783.

[5] Lei Xiaoen et al., Proceedings of ICBA, held at Lausanne, Switzerland, 1987.

[6] Lei Xisoen et al,, Proceedings of $80 \mathrm{th}$ APCA Annual Meeting, New York, 1987. 